\section{Scales: A Turbo Pascal 6.0 Unit for Presenting Simple Attitude Scales}

The Turbo Pascal 6.0 unit, Scales, provides single-line commands for the presentation of a generic rating scale, and appropriate anchors for Likert-type scales, semantic differentials, and true-false scales. This software affords researchers a versatile and relatively simple means of incorporating rating and attitude scales into their own Pascal programs. The Scales unit (written to be used in text mode) contains 17 different features, each of which can be used independently or jointly with each other.

The primary feature of the unit is the function rating scale. By providing appropriate parameters, this function will present a generic rating scale on the computer screen and return an integer value corresponding to the respondent's choice. Researchers have the freedom to specify scale coordinates, text and background colors, and the number of scale points (up to 12). Responses are made by moving a box on the screen via right- and leftarrow keypresses. A boolean parameter allows experimenters to permit respondents to skip questions, or to require respondents to answer each question without skipping.

Three procedures in the Scales unit easily allow anchors to be placed at the endpoints of the scale. Two of these-likert_scale and semantic_differential_-anchor the scale with "strongly agree" and "strongly disagree," and specified word pairs, respectively. By providing the same coordinates and scale size that were used in the rating_scale function, the anchors are automatically adjusted to the scale. The procedure true false assumes that the scale size is 2 , and anchors the 2 points with the words true and false. Each of these three procedures contains parameters to allow counterbalancing of the anchors.
Three of the remaining procedures display text in specified colors, either at specified $x$ and $y$ coordinates (LineWrite) or centered horizontally at specified $y$ coordinates (center_write, HeadFoot). Three other procedures assist in the description of the rating scale usage (right arrow_key, left_arrow_key, scale_instructions). The final procedure, border_window, employs Turbo Pascal's window procedure in specifying an active window and places a specified character border around this window.

Of the six remaining functions, four return specific values from the keyboard (get_char, get_integer, get_real, get_string). The boolean function turn the_page presents the instruction "Press $\mathrm{C}$ to continue" at specified coordinates and returns a value of true and clears the screen when $\mathrm{C}$ is pressed. The boolean function "yes" returns a value of true if a $\mathrm{Y}$ is pressed, and a value of false if an $\mathrm{N}$ is pressed.

A sample of 60 subjects completed an hour-long computer-administered questionnaire employing the Scales unit features. In the final question, the subjects were asked how easy the computer rating scales were to use. On a 10-point scale (with 10 being very easy), the mean response was $8.03(S D=2.26)$. In general, they found these scales easy to use.

Availability. The Scales unit, its source code, full documentation, and an example program can be obtained from Michael Platow, Department of Psychology, University of Otago, P.O. Box 56, Dunedin, New Zealand. Please send an IBM formatted disk and $\$ 3.00$ (U.S.) for postage.

Michael J. Platow and Roger Shave University of Otago

(Manuscript received March 17, 1993; revision accepted for publication March 8, 1994.) 